\title{
MASS TRANSFER IN CORRUGATED-PLATE MEMBRANE MODULES. II. ULTRAFILTRATION EXPERIMENTS*
}

\author{
M.J. van der WAAL ${ }^{1}$, S. STEVANOVIC ${ }^{2}$ and I.G. RACZ ${ }^{1, * *}$ \\ ${ }^{1}$ University of Twente, Dept. of Chemical Engineering, P.O. Box 217, 7500 AE Enschede \\ (The Netherlands) \\ ${ }^{2}$ University of Beograd, Fac. of Technology and Metallurgy, Inst. of Analytical Chemistry, \\ P.O. Box 494, 11000 Beograd (Yugoslavia)
}

\section{Summary}

The application of corrugations as turbulence promoters in membrane filtration was studied. In ultrafiltration experiments with polysulfone membranes using Dextran T70 as solute, it was found that the corrugations result in reduced energy consumption or pressure drop compared with flat membranes at a given value of the flux through the membrane. There appears to be an optimal mutual distance between the corrugations.

\section{Introduction}

In Part I hyperfiltration experiments with corrugated membranes installed at one wall of a rectangular channel were described. From these experiments, mass-transfer coefficients were estimated. Despite experimental uncertainties the conclusion could be drawn that the application of corrugations to an originally flat membrane did not damage the membranes and improved the overall mass transfer coefficient, even with a reduced energy consumption for a given value of the mass transfer coefficient.

It is the purpose of the investigation described in this part to confirm the results from the hyperfiltration experiments by ultrafiltration experiments.

\section{Experimental}

\section{Corrugated plates}

The corrugated plates used for the investigation described in Part I are also used in this work.

\footnotetext{
*Paper presented at the Workshop on Concentration Polarization and Membrane Fouling, University of Twente, The Netherlands, May 18-19, 1987.

**To whom correspondence should be addressed.
} 


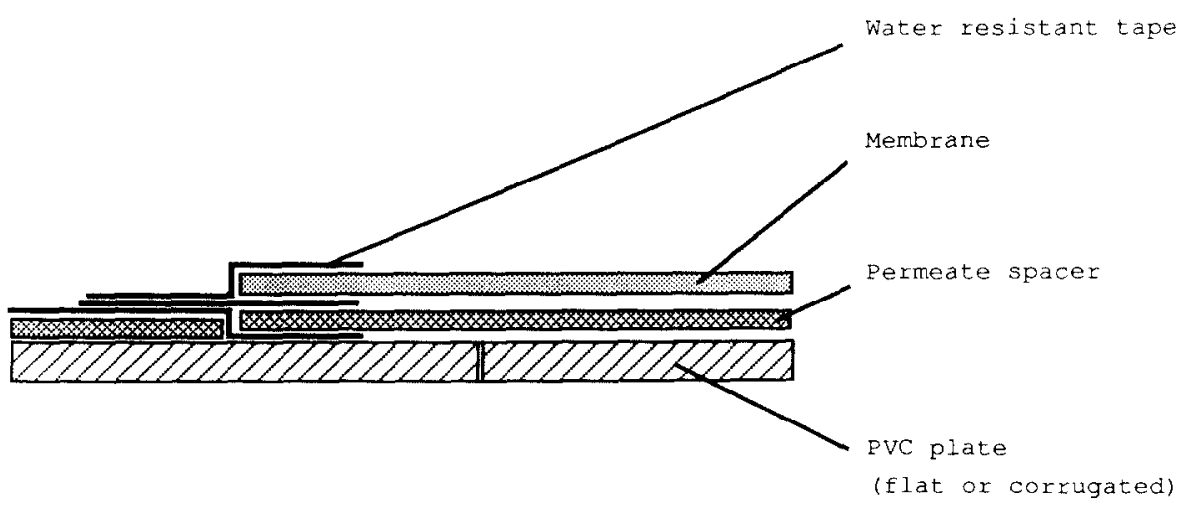

Fig. 1. Schematic of the membranc assembly in the module.

\section{Membranes}

Commercially available polysulfone membranes were used (Kalle PS-50, Nadir 66 and Nadir 47). The properties of Nadir 66 and Nadir 47 were the same within experimental accuracy. No description of the nominal properties of these membranes was received from the supplier.

\section{Modules and installation}

The modules and installation are the same as described in Part I.

\section{Mass transfer measurements}

The permeate flux through rectangular pieces of membrane was measured. Only part of the surface of the corrugated or flat plate was covered with a membrane, as for high fluxes the capacity of the feed pump was too low to reach the desired pressures of $800-1000 \mathrm{kPa}$. A sketch of the assembly of the membranes on the plate is given in Fig. 1. The masking of the membrane allowed measurement of the flux through an accurately known membrane area. Also on one plate two membranes of different type could be used at the same time.

After installing the membrane into the module, the apparatus was filled with water and pressurized. Then a feed flow velocity was set. A fresh membrane was conditioned for at least 16 hours at a minimum pressure of $600 \mathrm{kPa}$ before mass transfer measurements were performed.

Dextran T70 was used as the solute in water at a concentration of about 500 $\mathrm{mg} / \mathrm{l}(\mathrm{ppm})$ in most experiments. The concentration was determined by total organic carbon analysis.

\section{Theory}

For the description of the permeate flux in ultrafiltration two basically different models are given in the literature: the gel formation model [1] and the osmotic pressure model [2]. 
In the original gel formation model it is assumed that the solution to be filtered has a negligible osmotic pressure, even at the concentration near the membrane wall. When the pressure difference across the membrane is increased this concentration becomes so high that gelation or precipitation occurs. The gel or precipitate causes an extra resistance to solvent flow through the membrane. The layer of gel or precipitate will be so thick that the reduced convection of rejected solutes towards the membrane is balanced by back-diffusion of these solutes. The back-diffusion is assumed to be controlled by the mass-transfer coefficient and the difference in concentration in the feed flow and at the membrane wall. When gel formation occurs, the permeate flux can be described by [3]

$J=k \ln \frac{C_{\mathrm{g}}-C_{\mathrm{p}}}{C_{\mathrm{f}}-C_{\mathrm{p}}}$

where $k$ is the mass transfer coefficient, all $C$ s are concentrations of solute, and the subscripts $g, p$ and f correspond to gel, permeate and feed, respectively.

The osmotic pressure model assumes no gelation or precipitation, but states that the osmotic pressure near the membrane wall can become so high that the driving force and hence the flux through the membrane is greatly reduced. Especially in the case when the osmotic pressure is more than proportional to the solute concentration, Wijmans [2] deduced that for high pressures the permeate flux is independent of the pressure difference, and fully determined by the mass transfer coefficient. Wijmans [2] shows, using an exponential relationship between concentration and osmotic pressure

$\pi=a C^{n}$

that the flux at high pressure can become almost independent of the pressure. When the flux is independent of pressure, the derivative of flux versus the logaritm of feed concentration is equal to the value of the mass-transfer coefficient with a negative sign [2]. The pressure at which the flux can be considered to be independent of the pressure depends among other factors also on the membrane resistance. A low membrane resistance results in a low pressure for which this independency can be assumed. A higher resistance means high pressures should be applied to reach a pressure-independent flux. From the work of Wijmans, using eqn. (2), the flux $J_{1}$ can be calculated from:

$J_{1}=\frac{\Delta P-a C_{\mathrm{f}}^{n} \exp \left(n J_{1} / k_{1}\right)}{R_{\mathrm{m}}}$

at a given value of the mass transfer coefficient $k_{1}$. The rejection is taken to be $100 \%$. At the same pressure difference the fluxes at two values for $k$ can be compared:

$J_{1} R_{\mathrm{m}}+a C_{\mathrm{f}}^{n} \exp \left(n J_{1} / k_{1}\right)=\Delta P=J_{2} R_{\mathrm{m}}+a C_{\mathrm{f}}^{n} \exp \left(n J_{2} / k_{2}\right)$ 
which means that the applied pressure $\Delta P$ can be split in two parts, the first being the pressure drop over the "clean" membrane, the second being the osmotic pressure. In Fig. 2, a graphical impression of the system is given. If the resistance of the membrane is very low, so that

$J_{i} R_{\mathrm{m}} \ll a C_{\mathrm{f}}^{n} \exp \left(n J_{i} / k_{i}\right)$,

eqn. (4) can be simplified to:

$a C_{\mathrm{f}}^{n} \exp \left(n J_{1} / k_{1}\right)=a C_{\mathrm{f}}^{n} \exp \left(n J_{2} / k_{2}\right)$

from which follows:

$\frac{J_{1}}{J_{2}}=\frac{k_{1}}{k_{2}}$

This result is the same as for the gel formation model. It should be noted, however, that the similarity between both models with present-day membranes is only true at high pressure differences. For pressure differences where the flux still is a function of pressure, but deviates from the pure water flux,

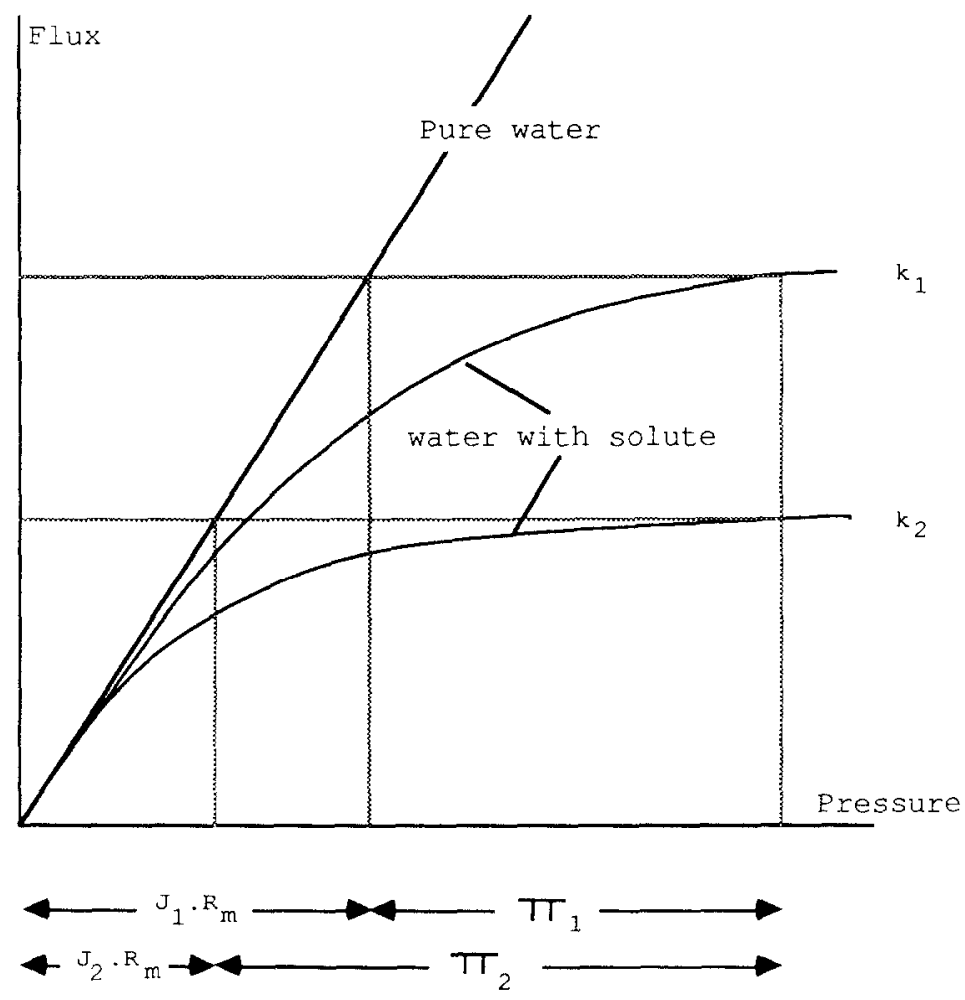

Fig. 2. Sketch of ultrafiltration flux as a function of pressure and mass transfer. 
the flux value is less than proportional to the value of the mass transfer coefficient; assuming $k_{1}>k_{2}$, meaning $J_{1}>J_{2}$, eqn. (3) and Fig. 2 yield:

$a C_{\mathrm{f}}^{n} \exp \left(n J_{1} / k_{1}\right)<a C_{\mathrm{f}}^{n} \exp \left(n J_{2} / k_{2}\right)$

from which follows:

$\frac{k_{1}}{k_{2}}>\frac{J_{1}}{J_{2}}$

provided the same value of $R_{\mathrm{m}}$ is used. From eqn. (9) it follows that the flux enhancement gives a pessimistic estimate of the improvement of the mass transfer coefficient.

\section{Results}

As in Part I all results described below are based on experiments performed in a channel of $6 \mathrm{~mm}$ height, $60 \mathrm{~mm}$ width and corrugations of half-cylinder shape with a height of $1.5 \mathrm{~mm}$ placed perpendicular to the main flow. Unless stated explicitely, the corrugations are only applied at the side where the membrane was used, the opposite side remaining flat.

\section{Deformability of the polysulfone membranes}

As can be seen from Figs. 3 and 4, the rejection of the polysulfone membranes is not influenced within experimental accuracy after deformation by the corrugations. For the Nadir 66 membrane the rejection at $800 \mathrm{kPa}$ is found to be between 85 and $100 \%$ for both flat and corrugated membranes, depending on the flow velocity.

\section{Mass transfer}

For a flat membrane the flux and rejection as a function of pressure differences and feed flow are given in Fig. 3. The shapes of the curves are representative for results from experiments using flat and corrugated polysulfone membranes and Dextran T70. Not only the flux, but also the rejection is influenced markedly by pressure and velocity. From the shape of the curves it can be concluded that the gel formation model is not applicable. No sharp change in the curve of flux versus pressure (which is expected for the gel formation model) is found whereas the flux also is still a slight function of pressure at high pressure. With some care the osmotic pressure model can be applied. As Dextran T70 is not a monodisperse material, $\left(M_{\mathrm{w}} 70,300 ; M_{\mathrm{n}} 36,200\right)$, part of the Dextran might pass the membrane unhindered, part will be partially rejected, and the remainder is fully rejected. This phenomenon also attributes to a possible discrepancy between fluxes and mass-transfer coefficients.

Distinct rejection curves are found for the PS-50 membrane on the one hand, and for Nadir 66 and Nadir 47 membranes on the other. For a flat membrane 
Rejection (8)
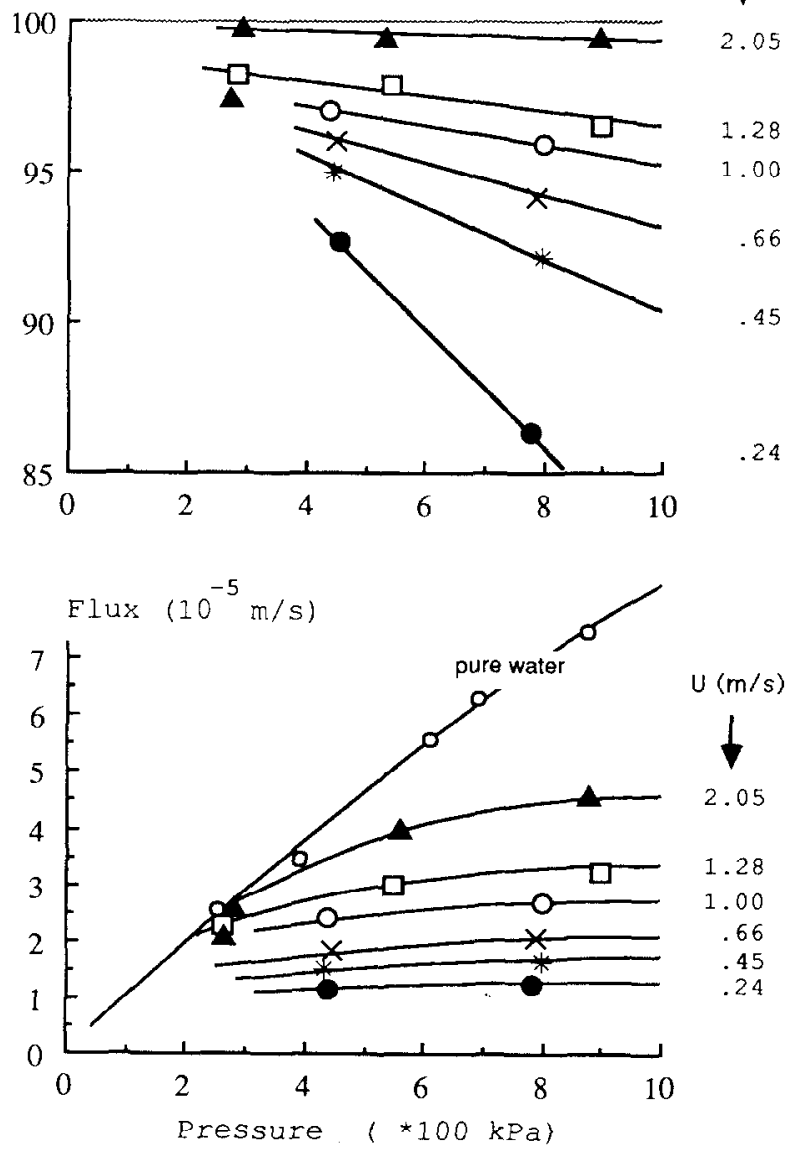

Fig. 3. Flux and rejection as a function of pressure and feed flow velocity for Nadir 66 flat membrane and Dextran T70 solute. Channel dimensions: height $6 \mathrm{~mm}$, width $60 \mathrm{~mm}$. Concentration of Dextran T70: $500 \mathrm{ppm}$.

at $800 \mathrm{kPa}$, however, the same curve of the flux versus flow velocity is found for all three membrane types. From this result it is concluded that for the membranes and solute studied here the experimental fluxes may be compared without taking rejection data into account.

Values of fluxes and rejections measured at $800 \mathrm{kPa}$ for corrugated membranes are given in Fig. 4. It is seen that the application of corrugations increases the flux by up to $150 \%$, depending on the mutual distances between the corrugations and on the flow velocity. In Fig. 5a, the flux values at $800 \mathrm{kPa}$ for a mutual distance of $40 \mathrm{~mm}$ are compared with the values for a flat membrane 

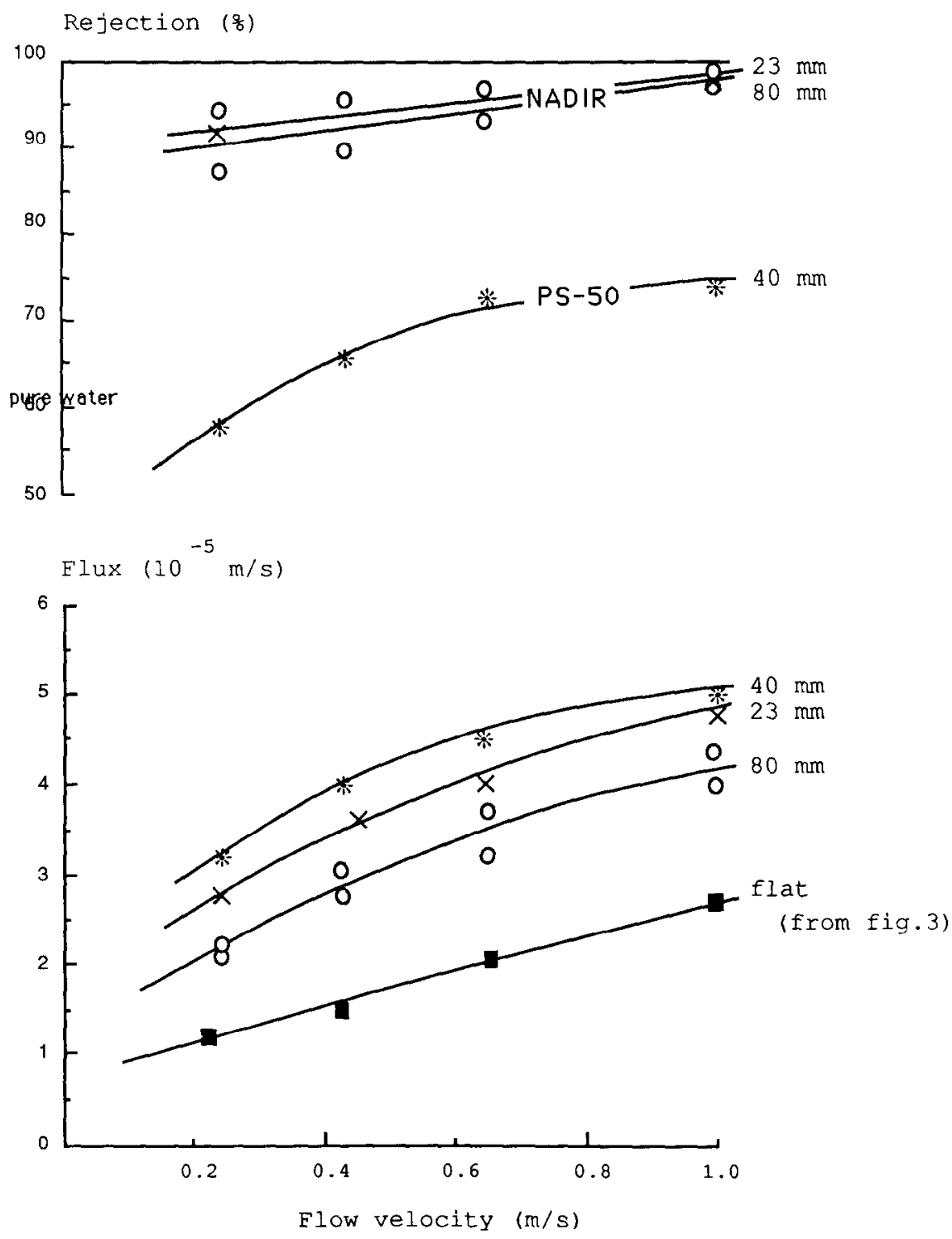

Fig. 4. Flux and rejection at $800 \mathrm{kPa}$ as a function of feed flow velocity for corrugated membranes and Dextran T70 solute. Concentration Dextran T70: 500 ppm.

at three velocities and three concentrations. For a flow velocity of $0.67 \mathrm{~m} / \mathrm{sec}$, flux values at three concentrations for mutual distances of 23,40 and $80 \mathrm{~mm}$ are given in Fig. 5b. At this velocity the data for the three feed concentrations can be represented by a straight line in a plot of flux versus $\ln$ (feed concentration), as suggested by the gel formation model and the osmotic pressure model 
of Wijmans [2]. No common concentration is found for which the flux is expected to become zero. The curves in Figs. $5 \mathrm{a}$ and $\mathrm{b}$ show that the increase in flux of corrugated membranes is not limited to a small concentration range.

It should be noted that the above results are for corrugations pressed into the membrane, the opposite side of the channel being flat. It appeared worthwhile to investigate the influence of corrugations at the opposite side of a membrane, which now is flat. The corrugations were glued on a perspex plate of 1.1 $\mathrm{mm}$ thickness, thus reducing the channel height. To elucidate the influence of channel height on mass transfer, several flat perspex plates with different heights were attached to the non-membrane wall. The corresponding fluxes at $800 \mathrm{kPa}$ are plotted in Fig. 6. Surprisingly, the flux is only markedly influenced for a channel height of $2.6 \mathrm{~mm}$, other channel heights showing no influence.

When corrugations are applied to the non-membrane wall, the curves given in Figs. 7 and 8 are found for channel heights of $4.9 \mathrm{~mm}$ and $3.8 \mathrm{~mm}$. From

$$
\text { FIux }\left(10^{-5} \mathrm{~m} / \mathrm{s}\right)
$$

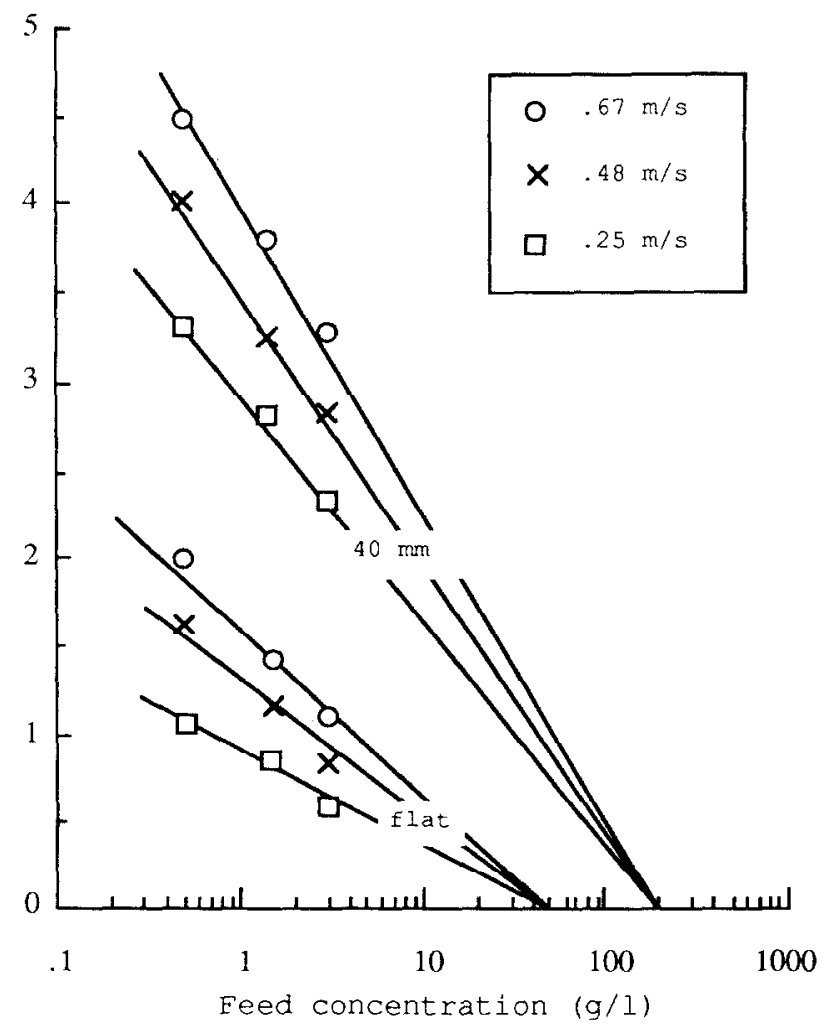

Fig. 5a. Flux as a function of concentration of Dextran T70 in the feed for a flat and a corrugated membrane at three flow velocities. Channel dimensions: height $6 \mathrm{~mm}$, width $60 \mathrm{~mm}$. 


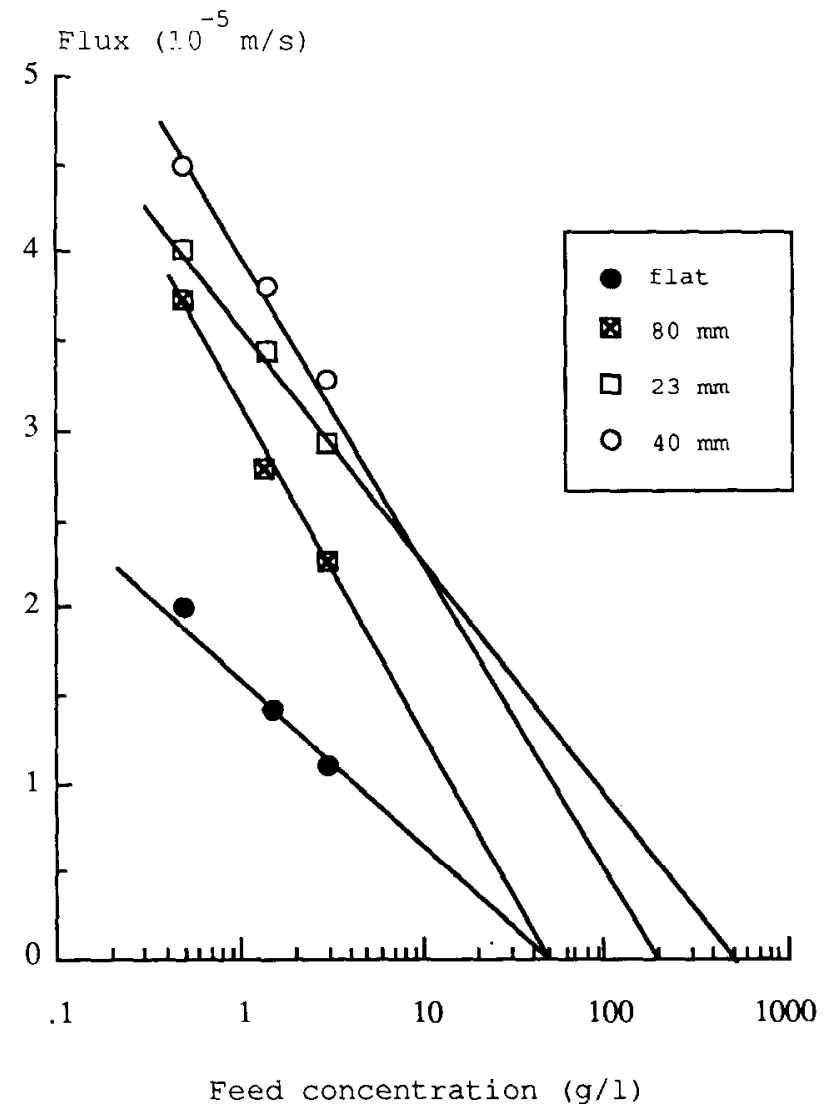

Fig. 5b. Flux as a function of concentration of Dextran T70 in the feed for flat and corrugated membranes at a flow velocity of $0.67 \mathrm{~m} / \mathrm{sec}$. Channel dimensions: height $6 \mathrm{~mm}$, width $60 \mathrm{~mm}$. Corrugations: half cylinders, height $1.5 \mathrm{~mm}$.

these figures it can be seen that the corrugations also positively influence the mass transfer at the opposite flat wall, the influence being stronger for reduced channel height. The mass transfer at the flat side, however, is still lower than at the corrugated side. This is seen in Fig. 9 for corrugations with a mutual distance of $40 \mathrm{~mm}$. The flux for a corrugated membrane is $50-100 \%$ higher than for a flat membrane with corrugations at the opposite wall.

Consumption of energy as a function of the flux at $800 \mathrm{kPa}$ with flat and corrugated membranes

As explained above, it is not possible to calculate exact values for the masstransfer coefficient from flux measurements, but the flux values can be used as a criterion for increase or decrease of the mass transfer. In fact, in membrane filtration one is mainly interested in flux and rejection, whereas mass transfer 


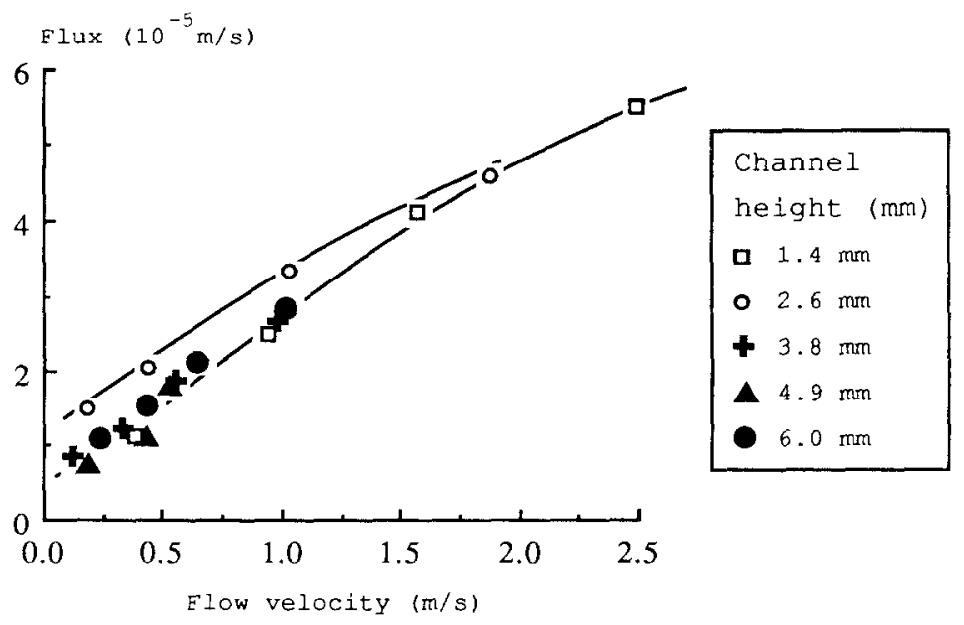

Fig. 6. Flux as a function of feed flow velocity at $800 \mathrm{kPa}$ for a flat membrane channel at different channel heights. Channel width: $60 \mathrm{~mm}$. Feed concentration: $500 \mathrm{ppm}$ Dextran T70 in water.

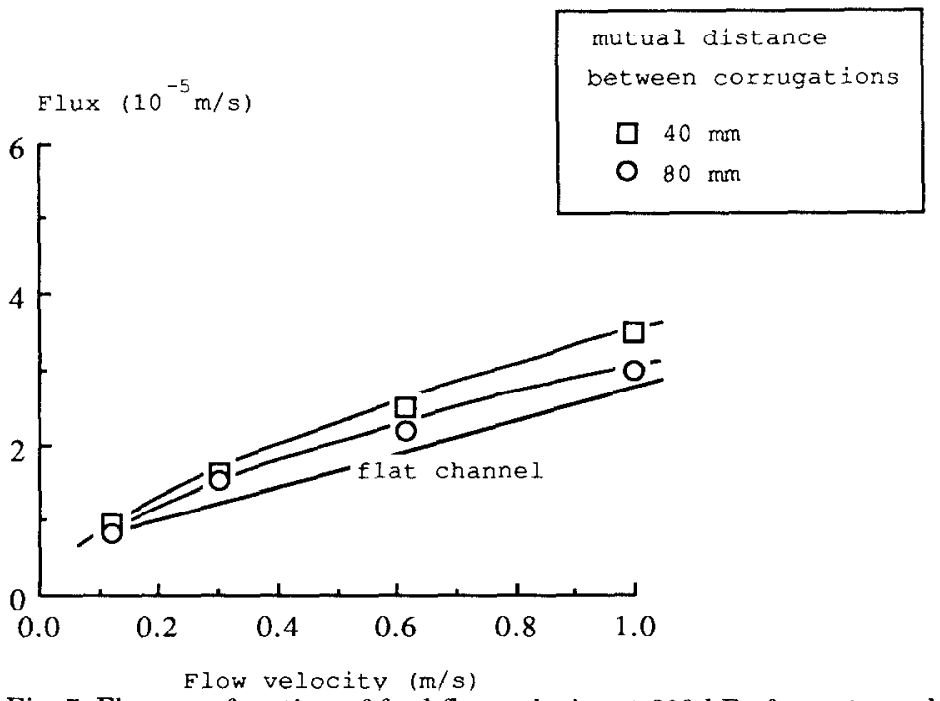

Fig. 7. Flux as a function of feed flow velocity at $800 \mathrm{kPa}$ for a chanıel height of $4.9 \mathrm{~mm}$ and a corrugated wall at the opposite side of the membrane. Channel width: $60 \mathrm{~mm}$. Feed concentration: 500 ppm Dextran T70 in water.

is "only" a determining factor besides membrane properties. Above, experimental fluxes are given for different flow velocities and geometries. In Part I friction factors are given, from which the pressure drop and energy consumption can be calculated. In Fig. 10 the flux is given as a function of the energy consumption for corresponding velocities along the membrane. In this figure results are given for membranes applied at the corrugated side of the channel, 


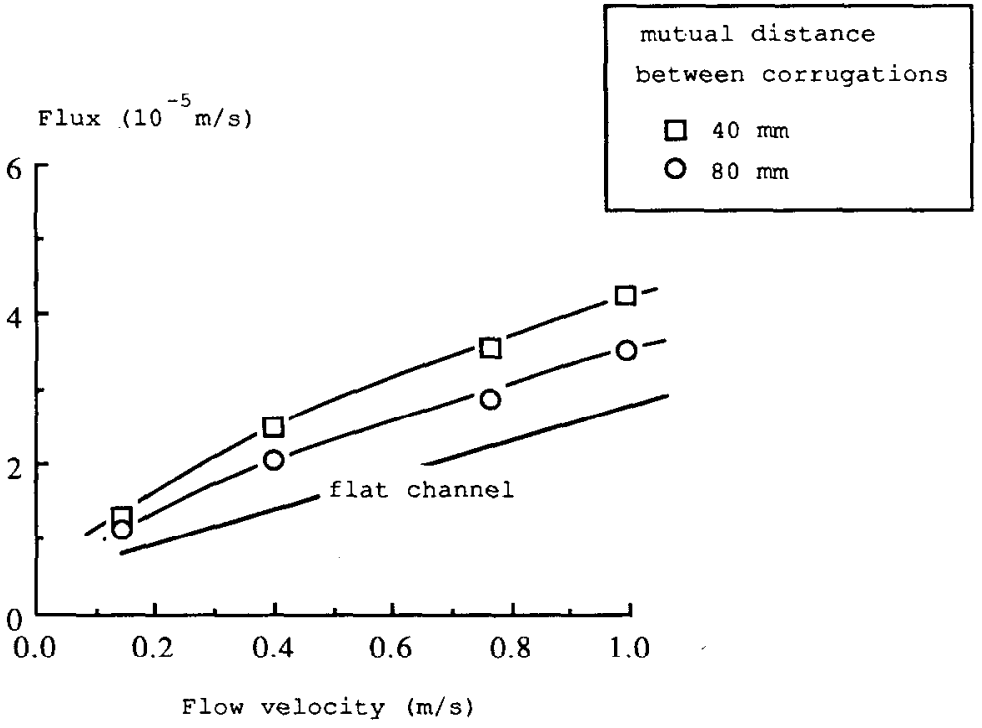

Fig. 8. Flux as a function of feed flow velocity at $800 \mathrm{kPa}$ for a channel height of $3.8 \mathrm{~mm}$ and a corrugated wall at the opposite side of the membrane. Channel width: $60 \mathrm{~mm}$. Feed concentration: $500 \mathrm{ppm}$ Dextran T70 in water.

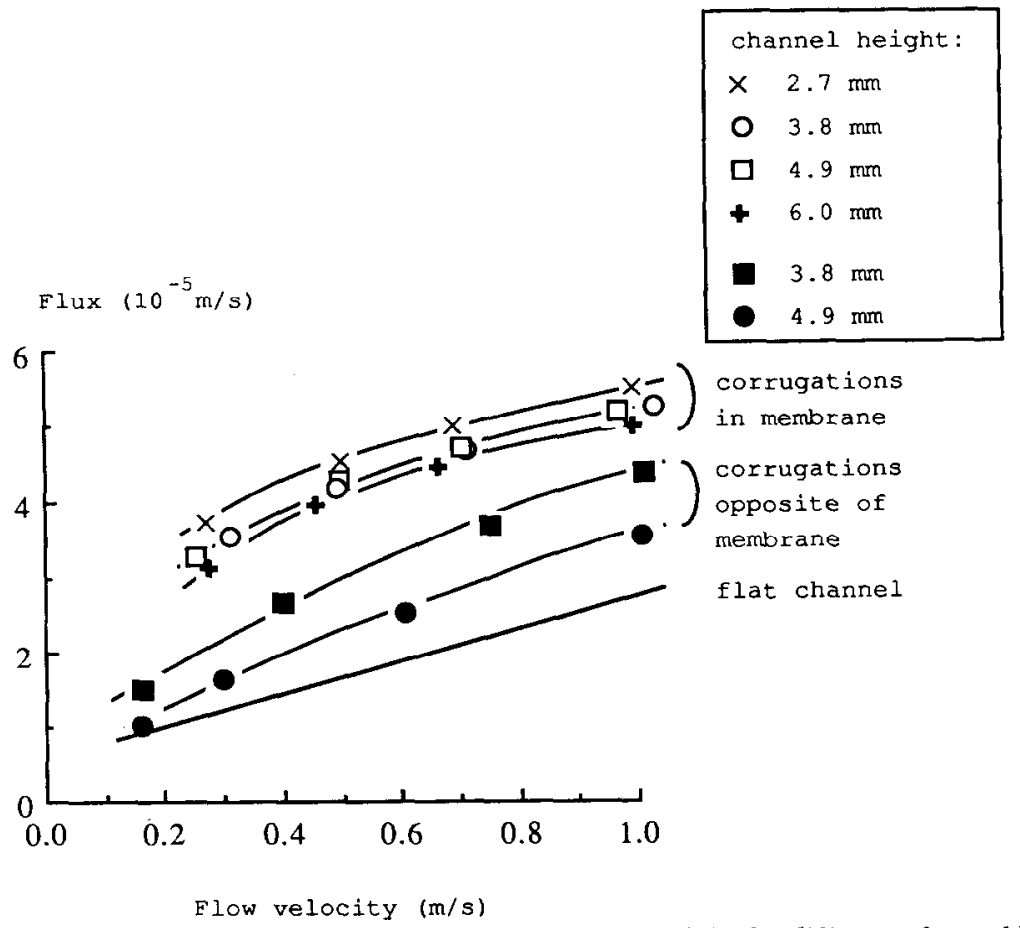

Fig. 9. Flux as a function of feed flow velocity at $800 \mathrm{kPa}$ for different channel heights and corrugations in the membrane and at the opposite side of the membrane. Channel width: $60 \mathrm{~mm}$. Feed concentration: $500 \mathrm{ppm}$ Dextran T70 in water. Corrugations: half cylinders, height $1.5 \mathrm{~mm}$, mutual distance $40 \mathrm{~mm}$. 
Flux $\left(10^{-5} \mathrm{~m} / \mathrm{s}\right)$

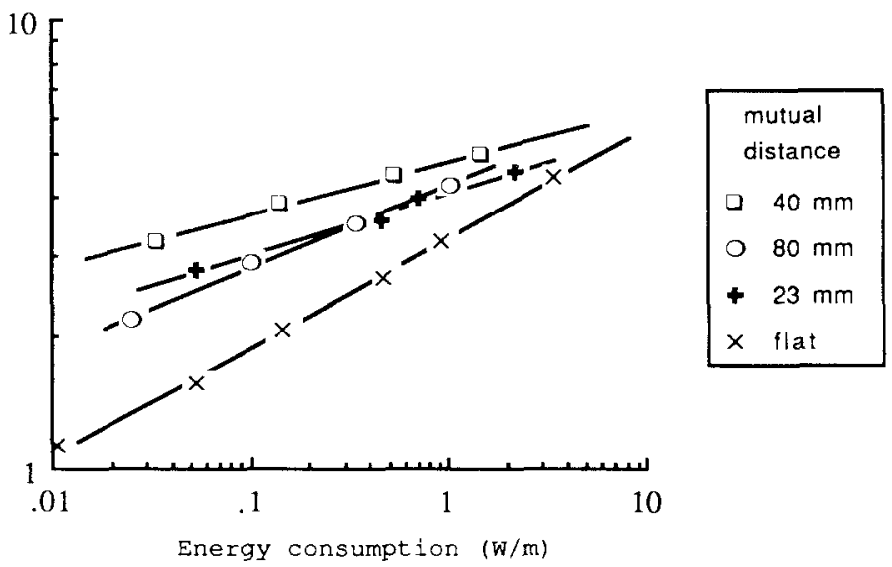

Fig. 10. Flux at $800 \mathrm{kPa}$ as a function of energy consumption for flat and corrugated membranes. Feed concentration: 500 ppm Dextran T70, half-cylindrical corrugations, height $1.5 \mathrm{~mm}$.

the corrugations being half cylinders with a height of $1.5 \mathrm{~mm}$. The figure can be compared with Fig. 9 in Part I, where for the same geometries the masstransfer coefficient is given as a function of the energy consumption. It is seen from Fig. 10 that the plate with corrugations at a mutual distance of $40 \mathrm{~mm}$ is the most effective one. As in Part I, all three plates (with mutual distances of 23,40 and $80 \mathrm{~mm}$ ) are more energy efficient than a flat plate within the range of velocities used. The figure shows a tendency for a reduced energy effectiveness at higher flow velocities. Extrapolated lines for a flat plate and the plate with corrugations at a mutual distance of $40 \mathrm{~mm}$ intersect at a flux of $7 \times 10^{-5}$ $\mathrm{m} / \mathrm{sec}$ and an energy consumption of $29 \mathrm{~W} / \mathrm{m}$, corresponding to a velocity of $4.4 \mathrm{~m} / \mathrm{sec}$ for a flat membrane in a channel of $6 \mathrm{~mm}$ height.

Pressure drop as a function of the mass-transfer coefficient with flat and corrugated plates

In part I, it was found that for a value of the mass-transfer coefficient of $4 \times 10^{-5} \mathrm{~m} / \mathrm{sec}$ the pressure drop for some corrugated plates was lower than for a flat plate. The calculation was done at only one value of the mass-transfer coefficient. Experimental uncertainties prohibited very accurate determination of the values of the mass-transfer coefficient. In the ultrafiltration experiments described in this part, fluxes as a result of a given flow velocity (and therefore mass transfer coefficient) are sufficiently accurate to study the flux as a function of pressure drop. The results are given in Fig. 11, from which it is concluded that for not too high flow velocities the corrugated plates are also advantageous as regards pressure drop.

The lines for the flat plate and the plate with corrugations having mutual 


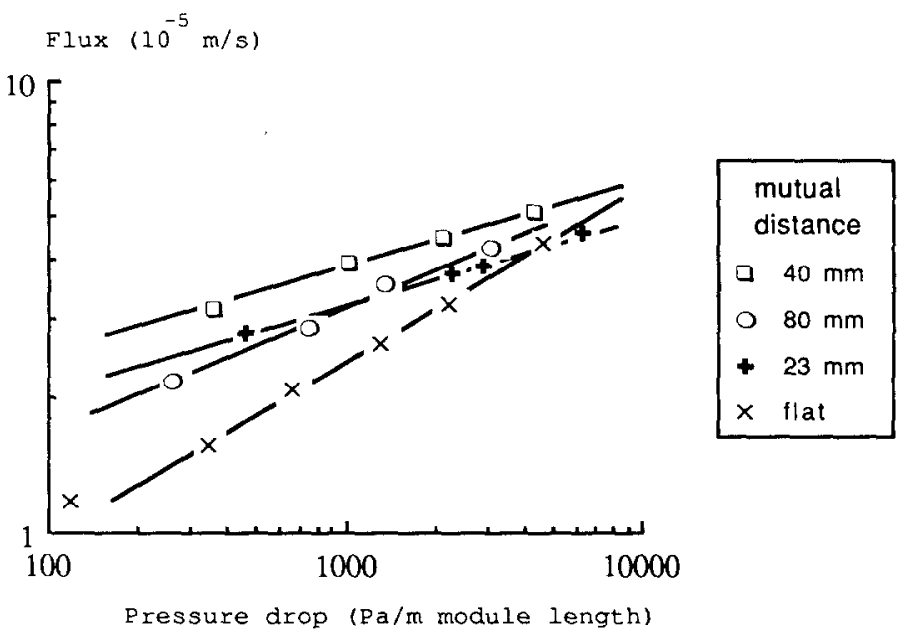

Fig. 11. Flux at $800 \mathrm{kPa}$ as a function of pressure drop for flat and corrugated membranes. Feed concentration: $500 \mathrm{ppm}$ Dextran T70, half-cylindrical corrugations, height $1.5 \mathrm{~mm}$.

distances of $40 \mathrm{~mm}$ intersect at a flux of $6 \times 10^{-5} \mathrm{~m} / \mathrm{sec}$ and a pressure drop of $11,000 \mathrm{~Pa} / \mathrm{m}$, corresponding to a velocity of $3.3 \mathrm{~m} / \mathrm{sec}$. This result means that corrugated plates are not only suitable for application in recirculating systems, but also in once-through systems.

\section{Discussion}

In Part I the results of the hyperfiltration experiments are discussed and partially explained with the help of literature from other research fields. In this part ultrafiltration experiments are described resulting in more accurate values for the flux, which are mainly determined by the values of the masstransfer coefficient. The conclusion from Part I that corrugated membrane walls can be energetically effective is confirmed, whereas here the additional conclusion can be drawn that application of corrugated plates can result in a lower pressure drop for the same flux values. As for the energy-effectiveness, the latter conclusion can be explained by the increased local turbulence, as determined by Hijikata and Mori [4]. Turbulence is a cause of energy consumption and pressure drop. If turbulence can be generated at a desired intensity only in a small layer near the wall where the mass transfer should take place, energy is - almost - only dissipated in this small layer. In that case, the liquid far from the wall, not active in mass transfer, is inactive or at least on a low level in dissipating energy or generating pressure drop.

From Figs. 10 and 11 it can be seen by extrapolation that the advantage of the corrugated plates ceases to exist at high velocities. Also, the curves for the plate with corrugations at a mutual distance of $80 \mathrm{~mm}$ are more parallel to 
those for the flat plate than are the plates with corrugations at smaller mutual distances. The latter phenomenon is explained by the limited area behind the corrugation which is influenced by the presence of the corrugation resulting in locally high values of mass transfer. Turbulence induced by the corrugation mixes with the main stream and most of it is dissipated after some distance. The first phenomenon (reduced effectiveness at high velocities) may be explained by the following hypothesis: If the level of turbulence in the circulation area behind the corrugation is lower than in the main stream, the increased level of turbulence around the point of reattachment of the boundary layer to the wall might not be able to compensate for the reduced level in the circulation area. As nothing is known at this moment about the magnitude of reduction or enhancement of the level of turbulence, this hypothesis is only preliminary.

\section{Conclusions}

From hyperfiltration and ultrafiltration experiments it can be concluded that the application of membranes pressed over corrugations of half cylindrical shape results in reduced energy consumption or pressure drop. There appears to be an optimal mutual distance between the corrugations.

\section{List of symbols}
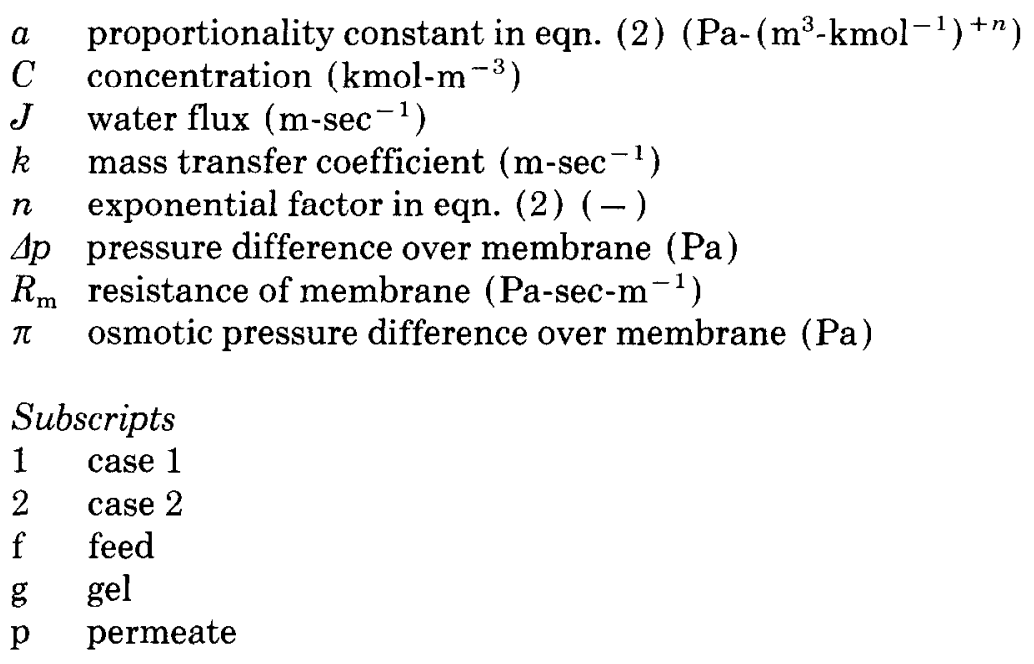

\section{References}

1 W.F. Blatt, A. Dravid, A.S. Michaels and L. Nelsen, Solute polarization and cake formation in membrane ultrafiltration: Causes, consequences, and control techniques, in: J.E. Flinn (Ed.), Membrane Science and Technology, Plenum Press, New York, NY, 1970, p. 47. 
2 J.G. Wijmans, S. Nakao and C.A. Smolders, Flux limitation in ultrafiltration: Osmotic pressure model and gel layer model, J. Membrane Sci., 20 (1984) 115.

3 P.L.T. Brian, Mass transport in reverse osmosis, in: U. Merten (Ed.), Desalination by Reverse Osmosis, MIT Press, Cambridge, MA, 1968, p. 161.

4 K. Hijikata and Y. Mori, Fundamental study of heat transfer augmentation of tube inside surface by cascade smooth surface-turbulence promoters, Wärme- Stoffübertrag., 21 (1987) $115-124$. 